\title{
The value of contrast-enhanced FLAIR magnetic resonance imaging in detecting meningeal abnormalities in suspected cases of meningitis compared to conventional contrast-enhanced T1WI sequences
}

\author{
Wael Hamza Kamr ${ }^{1 *}$, Mohamed Gaber Eissawy ${ }^{2}$ and Amr Saadawy ${ }^{3}$
}

\begin{abstract}
Background: Early diagnosis of meningitis with magnetic resonance imaging (MRI) would be useful for appropriate and effective management, decrease morbidity and mortality, and provide better diagnosis and treatment. The objective of the current study is to compare the accuracy of contrast-enhanced FLAIR (CE-FLAIR) and contrastenhanced T1WI (CE-T1WI) in the detection of meningeal abnormalities in suspected cases of meningitis.

Results: Out of 45 patients, 37 patients were confirmed to have meningitis on CSF analysis. Out of the 37 patients, 34 patients were positive on CE-FLAIR sequence and 27 were positive on CE-T1WI. The sensitivity of CE-FLAIR sequence was $91.9 \%$ and specificity 100\%, while the sensitivity of CE-T1WI sequence was $73 \%$ and specificity $100 \%$.

Conclusion: CE-FLAIR is more sensitive than CE-T1WI in diagnosis of meningitis. It is recommended to be used in any cases with clinically suspected meningitis.
\end{abstract}

Keywords: MRI T1 contrast, Contrast FLAIR, Meningitis

\section{Background}

Meningitis is a serious disease worldwide. It can be a fatal disease if not properly managed [1]. Clinical and laboratory evaluation can establish the diagnosis of meningitis. Evaluation of cerebrospinal fluid (CSF) is the most critical component of meningitis diagnosis [2].

In the diagnosis of meningitis, computed tomography (CT) and MRI may have major roles, but MRI is much more reliable and should be considered as the first-line imaging modality in brain infections $[1,3]$.

\footnotetext{
* Correspondence: dr.waelkamr@gmail.com

'Department of Diagnostic and Intervention Radiology, Faculty of Medicine,

Mansoura University, El Gomhorya St., Mansoura 35111, Egypt

Full list of author information is available at the end of the article
}

Contrast-enhanced MRI (CE-MRI) is also superior to contrast-enhanced CT to locate the meningeal affection and its complications. MRI is also superior in detecting extra-axial fluid collections due to the lack of the skull bony artifacts. Contrast administration aids in recognizing the blood-brain barrier breakdown and helps in recognizing the disease process that may not be visible on CT [4].

The standard contrast-enhanced MR series is the contrast-enhanced T1WI (CET1WI). FLAIR is a special reverse pulse sequence that effectively voids CSF signals. Contrast-enhanced fluid-attenuated inversion recovery (CE-FLAIR) is a different MRI sequence. Both sequences are important imaging methods that can be used to diagnose meningitis and its complications such as empyema
Springer Open
(0) The Author(s). 2020 Open Access This article is licensed under a Creative Commons Attribution 4.0 International License, which permits use, sharing, adaptation, distribution and reproduction in any medium or format, as long as you give appropriate credit to the original author(s) and the source, provide a link to the Creative Commons licence, and indicate if changes were made. The images or other third party material in this article are included in the article's Creative Commons licence, unless indicated otherwise in a credit line to the material. If material is not included in the article's Creative Commons licence and your intended use is not permitted by statutory regulation or exceeds the permitted use, you will need to obtain permission directly from the copyright holder. To view a copy of this licence, visit http://creativecommons.org/licenses/by/4.0/. 
and abscess. The sensitivities determined in different studies are very variable, which may be due to varying degrees of inflammation and the etiology of meningitis [3].

Early diagnosis of meningitis with MRI would be useful for appropriate and effective management, decrease morbidity and mortality, and provide better diagnosis and treatment.

The objective of the current study was to compare the accuracy of contrast-enhanced T1WI (CE-T1WI) and contrast-enhanced FLAIR (CE-FLAIR) in detecting meningeal abnormalities in suspected cases of meningitis.

\section{Methods}

\section{Study design}

This study was a prospective comparative study to compare CE-FLAIR and CE T1WI results of MRI sequences correlated with CSF analysis.

\section{Sample size}

Forty-five patients with clinically suspected meningitis were examined over a period of 2 years. It was a crosssectional study of validation. The sampling technique used was nonprobability, purposeful type.

\section{Inclusion criteria}

Patients referred to the Radiology Department for contrast-enhanced brain MRI for clinically suspected meningitis were included in this current study.

\section{Exclusion criteria}

Every patient who had any contraindication to MRI or history of allergy to gadolinium was removed from the research.

\section{Location}

The study was carried out in the magnetic resonance section in the Radiology Department.

\section{Equipment}

In the present study, a 1.5 tesla (T) Siemens "Magnetom Avanto" was conducted from the vertex to the skull base.

\section{Examination method}

After describing the aim, protocol, and risk-benefit ratio of the study and approval of the ethical committee, informed consent was obtained from all patients.

\section{Contrast-enhanced MRI protocol}

The CE-T1WI imaging parameters were TR: 500, TE: 8.9, FOV: $220 \mathrm{~mm}$, slice thickness: $5 \mathrm{~mm}$, slice interval: $1.5 \mathrm{~mm}$, and acquisition time: $4 \mathrm{~min}$. The CE-FLAIR imaging parameters were TR: 9000, TE: 90, TI: 2500, FOV: $220 \mathrm{~mm}$, slice thickness: $5 \mathrm{~mm}$, slice interval: $1.5 \mathrm{~mm}$, and acquisition time: 2 min $15 \mathrm{~s}$. Intravenous gadolinium contrast medium is administered to all patients (the patient's weight determined the dose) at a rate of 0.2 $\mathrm{mL} / \mathrm{s}$ from a computer-controlled injector (Medrad).

Two experienced (more than 10-year experience) independent radiologists blinded to CSF results evaluated both MRI sequences (CE-FLAIR and CE-T1WI) through simple visual inspection with special emphasis to detect any meningeal abnormality. The exam was considered positive if the radiologist detected any abnormal meningeal enhancement.

CSF examination was done for all patients and was reported as positive or negative for diagnosing meningitis.

\section{Statistical analysis}

The data collected was analyzed and findings were obtained using the Statistical Package for Social Science (SPSS) windows package version 22.0. Descriptive analysis was conducted, i.e., frequencies and percentages for the continuous variables such as age for categorical variables such as gender, mean, and standard deviation. The significance level was considered if the $P$ value $<0.05$ was found. Sensitivity, specificity, and negative and positive predictive values were calculated by using CSF analysis as a standard of reference.

\section{Results}

Fifty patients with symptoms suspicious of meningitis during the recruitment period were sent for MRI examination. Five patients were removed from the study because 1 patient had metallic implants that were not MR compatible (cardiac pacemaker) and 4 patients were claustrophobic. Consequently, the total number of patients included in this study was 45 , who were exposed to MRI for clinically suspected meningitis.

Of 45 patients, $28(62.3 \%)$ were male patients and 17 $(37.7 \%)$ were females. In males, the mean age ranged from 25 to 75 years was $36.0 \pm 16.25$ years. The mean age in females ranging from 10 to 75 years was $44.75 \pm$ 12.68 years. Every patient was subjected to lumbar puncture for CSF analysis after the MRI exam to confirm the diagnosis. Among 45 patients, 37 patients $(82.2 \%)$ had positive results with CSF and the remaining 8 patients (17.8\%) had negative results.

The analysis of unenhanced routine brain images did not show any meningeal abnormality, but three patients showed abnormal sulcal bright signal on the pre-contrast FLAIR sequence.

In post-contrast sequences, abnormal meningeal enhancement was found in 27 patients in both CE-T1WI and CE-FLAIR sequences (Figs. 1 and 2). CE-FLAIR images allowed for differentiation between abnormal meningeal enhancement and the cortical veins (Fig. 3). Abnormal meningeal enhancement was seen in 7 cases in only CEFLAIR sequence (Figs. 4 and 5). So, in CE-T1WI images, 27 patients showed pathological meningeal enhancement while 18 patients had a negative study. In CE-FLAIR 


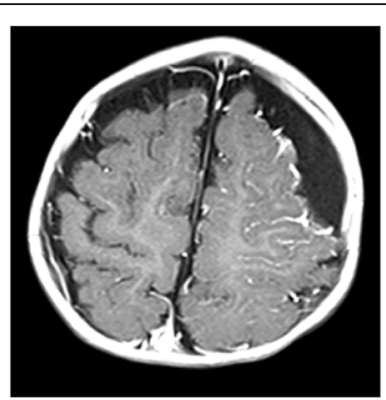

(A)

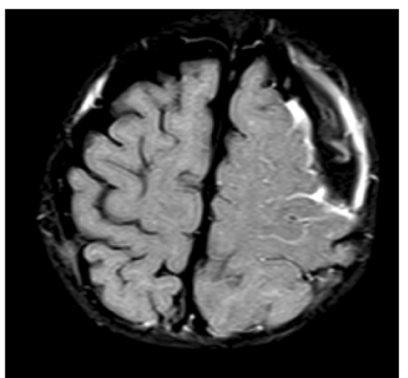

(B)

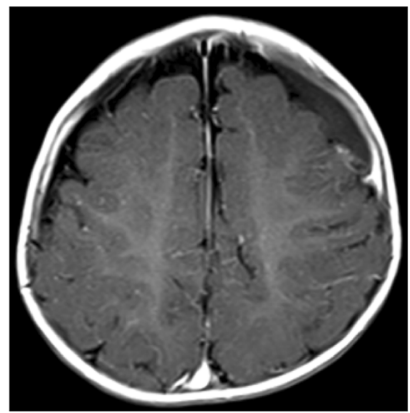

(D)

(E)

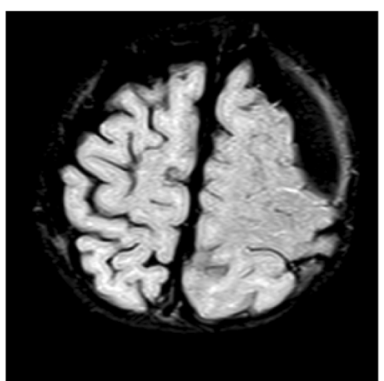

(C)

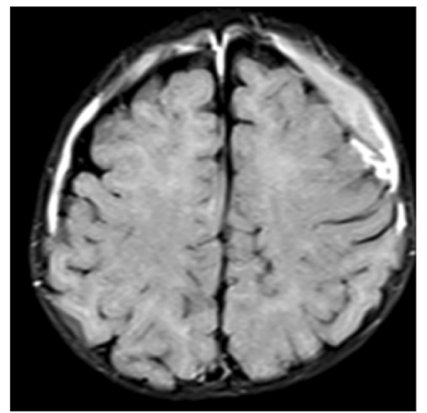

Fig. 1 Axial contrast-enhanced MRI T1WI (a) showing small high left frontal subdural fluid collection with related meningeal enhancement. Similar findings but more obvious are seen in axial contrast-enhanced FLAIR (b). The non-contrast FLAIR (c) shows the difference between pre-contrast study and post-contrast enhancement. On follow-up, contrast-enhanced T1WI (d) shows minimal meningeal enhancement which appears more obvious on contrast-enhanced FLAIR image (e)

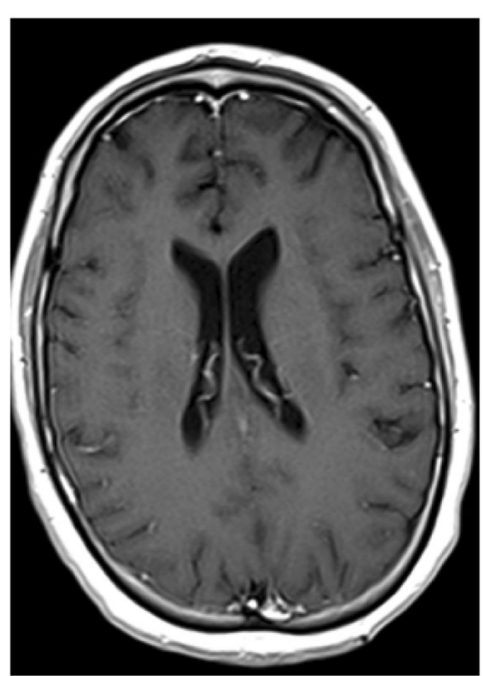

(A)

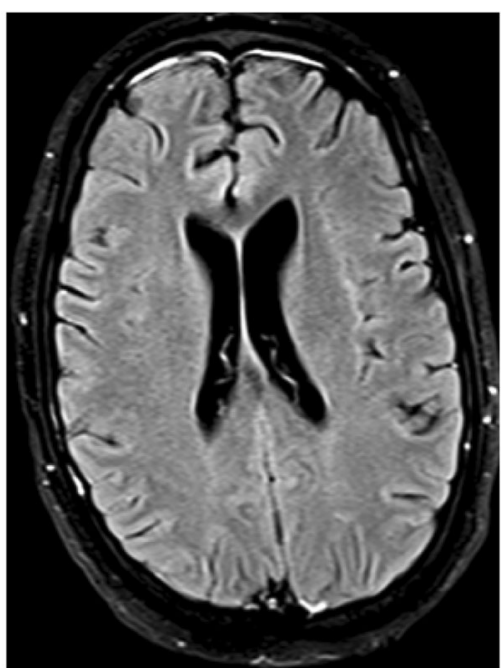

(B)

Fig. 2 Axial contrast-enhanced MRI, (a) T1 WI mild diffuse meningeal enhancement. Contrast-enhanced FLAIR (b) shows meningeal enhancement obvious mainly at both frontal regions 


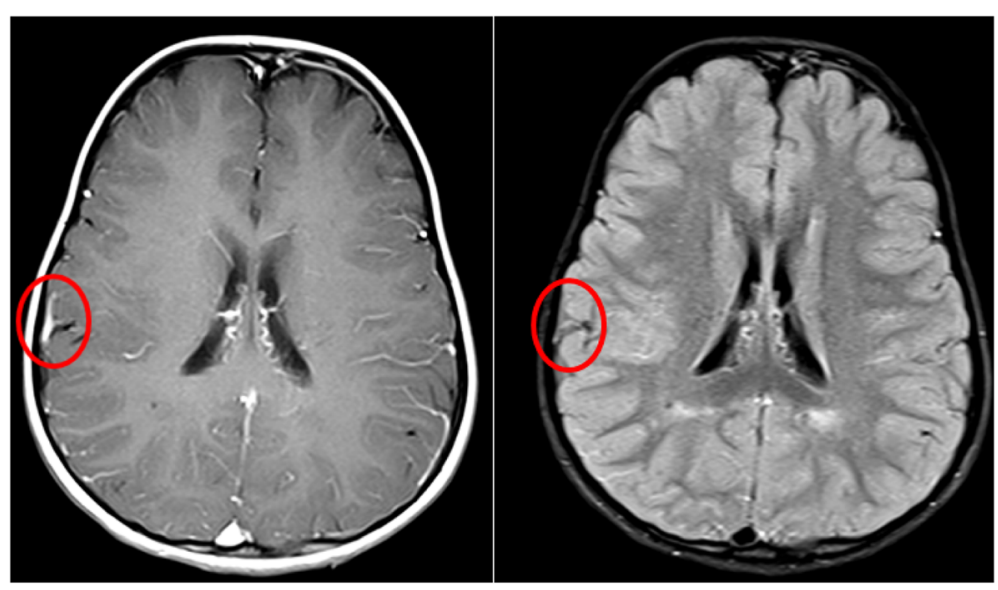

A)

B)

Fig. 3 a Axial contrast-enhanced T1WI MRI shows a small focal linear area of enhancement along the brain surface (red circle) that could represent focal meningeal enhancement versus enhancing cortical vein. b Axial contrast-enhanced FLAIR shows no enhancement in the corresponding area, reflecting that this enhancing area is cortical vein rather than true meningeal enhancement

images, 34 patients showed pathological meningeal enhancement while 11 patients had negative study (Table 1). Both radiologists reported the same findings in all cases with no detected mismatching in their interpretation.

The sensitivity of the CE-FLAIR sequences was $91.9 \%$, the specificity $100 \%$, the positive predictive value $100 \%$, and the negative predictive value $72.7 \%$, whereas the CET1WI showed a sensitivity of $73 \%$, a specificity of $100 \%$, a positive predictive value of $100 \%$, and a negative predictive value of $44.4 \%$ (Table 2).

\section{Discussion}

MRI plays an important role in diagnosing intracranial infections. MRI study may show many abnormalities, including a variable degree of abnormal meningeal contrast enhancement. The standard contrast-enhanced magnetic resonance series at most institutions is CE-T1WI. On
T1WI, however, meningeal enhancement is sometimes unnoticeable [5].

In detection of inflamed meninges, the CE-FLAIR sequence is superior to CE-T1WI. The meningeal disease can be visualized more effectively with CE-FLAIR images than it is in CE-T1WI because CE-FLAIR is more sensitive to lower contrast concentration as a result of its marked sensitivity to limited alteration of CSF composition $[6,7]$.

CE-FLAIR images also allow for more accurate differentiation between abnormal meningeal enhancement and the cortical veins [1]. CE-FLAIR demonstrates no enhancement of the cortical veins or normal meninges that can cause confusion with pathologically enhancing meninges on CE-T1WI.

In the current study, the sensitivity of the CE-FLAIR sequences was $91.9 \%$, the specificity $100 \%$, the positive predictive value $100 \%$, and the negative predictive value $72.7 \%$. The results of the current study are in accordance

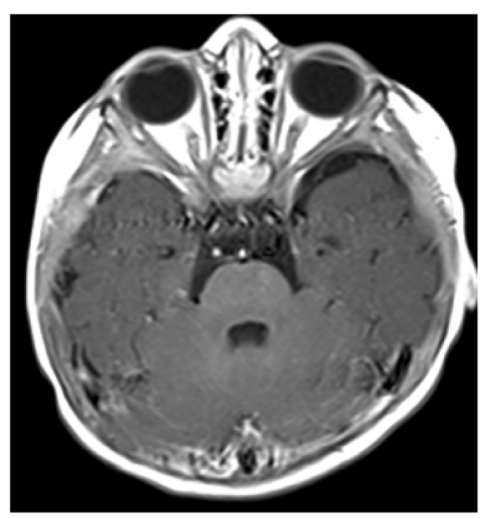

(A)

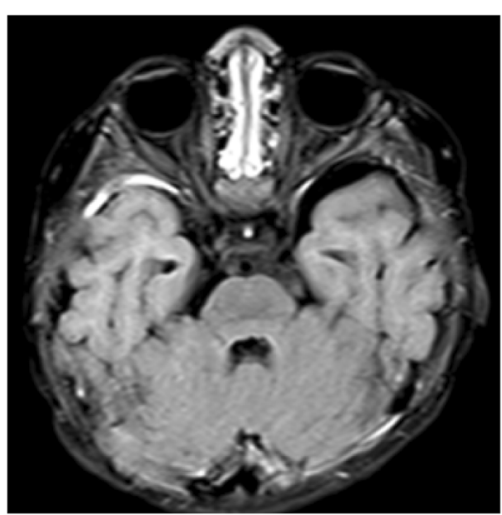

(B)

Fig. 4 Axial contrast-enhanced T1WI (a) shows no definite meningeal enhancement. Contrast-enhanced FLAIR (b) shows focal linear thick meningeal enhancement related to the right temporal lobe 


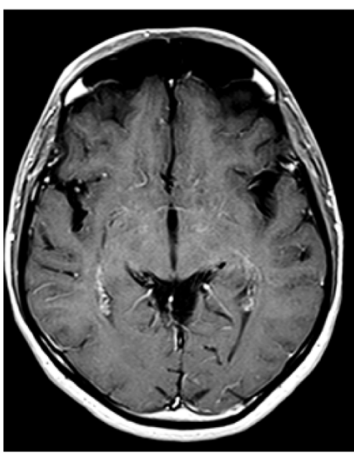

(A)

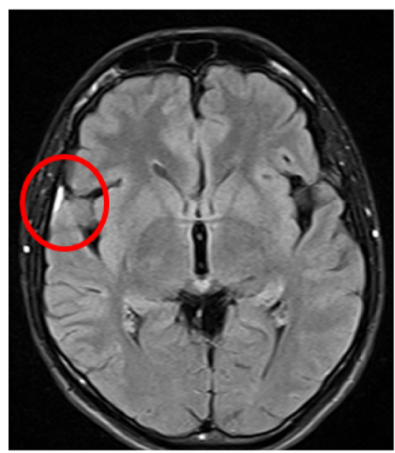

(B)

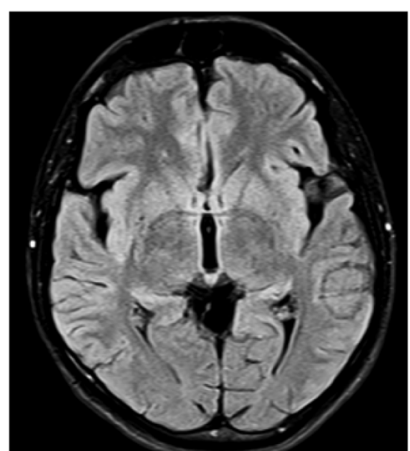

(C)

Fig. 5 Axial contrast-enhanced T1WI (a) shows no definite meningeal enhancement while contrast-enhanced FLAIR (b) shows small focal meningeal enhancement (red circle) related to the right temporal lobe compared to contrast-enhanced T1WI and non-contrast FLAIR (c)

with Mubasher [4], who stated that CE-FLAIR images had a sensitivity of $95.3 \%$ and CE-T1WI a sensitivity of $76.7 \%$, as well as Azad et al. [8], who noted that the CE-FLAIR images showed improved sensitivity, diagnostic accuracy, and better correlation with CSF compared to CE-T1WI sequences. Researchers concluded that the CE-FLAIR series is an appropriate modality to determine meningitis and could be included in the routine MR procedure.

In this study, the results of CE-FLAIR were compared to the results of CE-T1WI in confirming the diagnosis of meningitis and the final diagnosis was based on CSF results. No false positive outcomes were found in both CE-FLAIR and CE-T1WI. These results are approximately similar to Allesandra et al. [9], who studied the accuracy of MRI in early diagnosis of infectious meningitis with a focus on the importance of the series of contrast-enhanced FLAIR. There was confirmation of infectious meningitis in 12 patients. In all 12 patients, MRI CE-FLAIR showed abnormal meningeal enhancement, whereas CE-T1-WI was only positive in six cases. No false-positive or false-negative outcomes were found in CE-FLAIR sequences in their study. They concluded that if the CE-FLAIR sequence is used, MRI may play a crucial role in early screening for infectious meningitis.

In the current study, the CE-T1WI was routinely done without fat saturation. This may explain the difference in results compared to research done by Galassia et al. [10], who found that pathological meningeal enhancement was

Table 1 Results of MR in total cases $(n=45)$. CSF analysis was considered the gold standard (37 cases were positive and 8 cases were negative)

\begin{tabular}{lll}
\hline & CE-T1WI & CE-FLAIR \\
\hline True positive & 27 & 34 \\
False positive & 0 & 0 \\
True negative & 8 & 8 \\
False negative & 10 & 3 \\
\hline
\end{tabular}

seen in 35 patients in CE-T1WI with fat saturation and in 33 patients with CE-FLAIR. They found that, for the depiction of intracranial meningeal diseases, CE-T1WI with fat saturation is superior to CE-FLAIR sequence.

This study showed that CE-FLAIR has higher sensitivity yet equal specificity compared to CE-T1WI. This is in contrary to a study done in 2006, by Parmar and his colleagues [11] who published a study to evaluate CEFLAIR in the assessment of meningitis and proposed that CE-FLAIR has equal sensitivity but a higher specificity compared to CE-T1WI for diagnosing leptomeningeal enhancement.

Falzone et al. [12] conducted a study on CE-FLAIR versus CE-T1WI in brain imaging. They concluded the dominance of CE-FLAIR images compared to CE-T1WI in the identification of brain lesions. The findings of our analysis are comparable to their results, yet it is not possible to make an exact comparison as their study included other brain parenchymal lesions other than meningitis.

Although this study was focused on detecting pathological meningeal enhancement due to the inflammatory process, CE-FLAIR may be of value in detecting other causes of meningeal enhancement.

One study by Ercan et al. [13] reported that CE-FLAIR imaging is a valuable adjunct to CE-T1WI. Pre-contrast and contrast-enhanced FLAIR accurately detect leptomeningeal, cisternal, and cranial-nerve metastases [13]. Ultimately, due to its high sensitivity and specificity, the

Table 2 Results of MR in total cases $(n=45)$. CSF analysis was considered the gold standard (37 cases were positive and 8 cases were negative)

\begin{tabular}{lll}
\hline & CE-T1WI & CE-FLAIR \\
\hline Sensitivity & $73 \%$ & $91.9 \%$ \\
Specificity & $100 \%$ & $100 \%$ \\
PPV & $100 \%$ & $100 \%$ \\
NPV & $44.4 \%$ & $72.7 \%$ \\
\hline
\end{tabular}


findings of our study promote the use of CE-FLAIR sequence to confirm the diagnosis of meningitis.

\section{Conclusion}

MRI imaging of meningitis, CE-FLAIR sequence, is more accurate than the CET1WI sequence. Therefore, in suspected cases of meningitis, CE-FLAIR should be applied as a regular sequence for non-invasive diagnosis.

\section{Abbreviations}

MRI: Magnetic resonance imaging; FLAIR: Fluid-attenuated inversion recovery; CE-FLAIR: Contrast-enhanced fluid-attenuated inversion recovery; CET1WI: Contrast-enhanced T1weighted image; CSF: Cerebrospinal fluid; CT: Computed tomography; SPSS: Statistical Package for Social Science

\section{Acknowledgements}

Not applicable.

\section{Authors' contributions}

Guarantor of integrity of the entire study: W.H.K., A.S. Study concepts and design: WHK, AS Literature research: WHK, AS, MGE. Clinical studies: WHK, AS, MGE. Experimental studies/data analysis: WHK, AS, MGE. Statistical analysis: WHK, AS, MGE. Manuscript preparation: WHK, AS. Manuscript editing: WHK, AS. All authors read and approved the final manuscript.

\section{Funding}

Not applicable.

\section{Availability of data and materials}

The datasets used and/or analyzed during the current study available from the corresponding author on reasonable request.

\section{Ethics approval and consent to participate}

This study had approval from the Institutional Review Board of Security Forces Hospital, KSA.

Ethics committee's reference number is not available.

Written informed consent was obtained from all patients.

\section{Consent for publication}

All patients included in this research gave written informed consent to publish the data contained within this study. If the patient was less than 16 years old, deceased, or unconscious when consent for publication was requested, written informed consent for the publication of this data was given by their parent or legal guardian.

\section{Competing interests}

All authors declare that they have no competing interests.

\section{Author details}

${ }^{1}$ Department of Diagnostic and Intervention Radiology, Faculty of Medicine, Mansoura University, El Gomhorya St., Mansoura 35111, Egypt. ${ }^{2}$ Radiology Department, Minia University, Minia, Egypt. ${ }^{3}$ Radiology Department, Ain Shams University, Cairo, Egypt.

Received: 12 August 2020 Accepted: 2 November 2020

Published online: 16 November 2020

\section{References}

1. Vaswani AK, Nizamani WM, Ali M, Aneel G, Shahani BK, Hussain S (2014) Diagnostic accuracy of contrast-enhanced FLAIR magnetic resonance imaging in diagnosis of meningitis correlated with CSF analysis. ISRN Radiol 2014:578986

2. Pandey P, Jha B, Shrestha A (2015) Cytological and biochemical profile of cerebrospinal fluid from meningitis patients. Ann Clin Chem Lab Med 1(1):2-5

3. Lummel N, Koch M, Klein M, Pfister HW, Brückmann H, Linn J (2016) Spectrum and prevalence of pathological intracranial magnetic resonance imaging findings in acute bacterial meningitis. Clin Neuroradiol 26(2):159-167
4. Mubasher A (2018) Comparison of gadolinium based T1 weighted and flair Mr sequences for the assessment of leptomeningeal enhancement in meningoencephalitis. Int J Radiol 5(1):163-171

5. Lee EK, Lee EJ, Kim S, Lee YS (2016) Importance of contrast-enhanced fluidattenuated inversion recovery magnetic resonance imaging in various intracranial pathologic conditions. Korean J Radiol 17(1):127-141

6. Kim H-J (2014) Importance of contrast-enhanced fluid-attenuated inversion recovery imaging to detect paradoxical expansion of tuberculoma. Int J Infect Dis 24:37-39

7. Fukuoka $\mathrm{H}$ et al (2010) Comparison of the added value of contrastenhanced 3D fluid-attenuated inversion recovery and magnetizationprepared rapid acquisition of gradient echo sequences in relation to conventional postcontrast T1-weighted images for the evaluation of leptomening. Am J Neuroradiol 31(5):868-873

8. Azad R, Tayal M, Azad S, Sharma G, Srivastava RK (2017) Qualitative and quantitative comparison of contrast-enhanced fluid-attenuated inversion recovery, magnetization transfer spin echo, and fat-saturation T1-weighted sequences in infectious meningitis. Korean J Radiol 18(6):973

9. Splendiani A, Puglielli E, De Amicis R, Necozione S, Masciocchi C, Gallucci M (2005) Contrast-enhanced FLAIR in the early diagnosis of infectious meningitis. Neuroradiology 47(8):591-598

10. Galassi W, Phuttharak W, Hesselink JR, Healy JF, Dietrich RB, Imbesi SG (2005) Intracranial meningeal disease: comparison of contrast-enhanced MR imaging with fluid-attenuated inversion recovery and fat-suppressed T1weighted sequences. Am J Neuroradiol 26(3):553-559

11. Parmar H, Sitoh Y-Y, Anand P, Chua V, Hui F (2006) Contrast-enhanced flair imaging in the evaluation of infectious leptomeningeal diseases. Eur $J$ Radiol 58(1):89-95

12. Falzone C, Rossi F, Calistri M, Tranquillo M, Baroni M (2008) Contrast enhanced fluid-attenuated inversion recovery vs. contrast-enhanced spin echo T1- weighted brain imaging. Vet Radiol Ultrasound 49(4):333-338

13. Ercan N, Gultekin S, Celik H, Tali TE, Oner YA, Erbas G (2004) Diagnostic value of contrast-enhanced fluid-attenuated inversion recovery MR imaging of intracranial metastases. Am J Neuroradiol 25(5):761-765

\section{Publisher's Note}

Springer Nature remains neutral with regard to jurisdictional claims in published maps and institutional affiliations.

\section{Submit your manuscript to a SpringerOpen ${ }^{\circ}$ journal and benefit from:}

- Convenient online submission

- Rigorous peer review

- Open access: articles freely available online

High visibility within the field

- Retaining the copyright to your article

Submit your next manuscript at $\boldsymbol{\nabla}$ springeropen.com 